\title{
Oportunidades de Crecimiento y Estructura de Propiedad como Determinantes del Apalancamiento de las Empresas Españolas
}

\section{Growth opportunities and ownership structure as determinants of Spanish firms leverage}

\author{
Valentín Azofira Palenzuela *. Universidad de Valladolid \\ Paolo Saona Hoffmann. Universidad de Valladolid \\ Eleuterio Vallelado González **. Universidad de Valladolid
}

\begin{abstract}
RESUMEN Nuestro objetivo es analizar la decisión de endeudamiento de las empresas españolas, considerando los problemas de información asimétrica, los costes de agencia y la estructura de propiedad. Las hipótesis se establecen teniendo en cuenta el entorno institucional al que pertenecen las empresas. Para el análisis se utiliza un panel de datos incompleto de 111 empresas adquiridas durante los años 1991-2001. Los resultados obtenidos indican que: $i$ ) La elevada concentración accionarial de las empresas españolas favorece el uso de la deuda para financiar sus oportunidades de crecimiento; ii) Las empresas con déficit de fondos recurren preferentemente, a los recursos externos mientras cuenten con una estructura de propiedad mayoritaria; iii) Las empresas cotizadas mantienen una velocidad de ajuste a su ratio de endeudamiento óptima distinta a la de las empresas no cotizadas y a la de las empresas de otros países.
\end{abstract}

PALABRAS CLAVE Endeudamiento; Estructura de Propiedad; Oportunidades de Crecimiento; Entorno Institucional.

SUMMARY Our aim is analyse the debt decision of Spanish firms, considering the asymmetry information problems, the agency costs and the ownership structure. The hypotheses are evolved taking in account the institutional environment where the firms perform their activity. The analysis uses an unbalanced data panel of 111 firms for the period 1991-2001. Our main results are: $i$ ) The high ownership concentration of Spanish firms favour the use of debt to finance the growth opportunities; ii) The firms with deficit of funds have more access to external funds if they have a majority ownership structure, y iii) Spanish quoted firms adjust to their debt target ratio at a different speed than other Spanish non quoted firms or than firms from other countries.

KEY WORDS Debt; Ownership Structure; Growth Opportunities; Institutional Environment.

\section{INTRODUCCIÓN}

La relevancia de las decisiones financieras de las empresas está directamente relacionada con la consideración o no de las distintas imperfecciones que friccionan los mercados

${ }^{*}$ ) Los autores agradecen los comentarios y sugerencias recibidos de dos evaluadores anónimos.

$\left(^{\star \star}\right)$ Autor al que se deben remitir los comentarios y sugerencias sobre el trabajo: Eleuterio Vallelado González, Departamento de Economía Financiera y Contabilidad, Universidad de Valladolid, Avda. Valle de Esgueva, 6, 47011, Valladolid, España, Tel.: +34 983 423387, Fax: +34 983 183830, teyo@eco.uva.es. 
financieros. Tras las propuestas seminales de Modigliani y Miller (1958) bajo el supuesto de mercados de capitales completos, muy distintos trabajos han ampliado el análisis dando entrada a las imperfecciones de los mercados. En esta línea nuestro trabajo pretende analizar el endeudamiento de las empresas españolas considerando no sólo las asimetrías de información y los costes de agencia sino también su estructura de propiedad, la cual, a la luz del enfoque law\&finance, viene determinada por el sistema financiero por el grado de desarrollo de nuestra economía ${ }^{(1)}$.

La existencia de asimetrías de información y costes de agencia en las relaciones de endeudamiento genera los problemas de subinversión y sustitución de activos. Estos problemas son especialmente relevantes en aquellas empresas con oportunidades de crecimiento, elevadas tasas de endeudamiento y en aquellas cuya estructura de propiedad está muy concentrada. La suscripción de nuevos contratos de endeudamiento potencia estos problemas puesto que, por una parte, los accionistas querrán dejar pasar por alto determinados proyectos rentables de inversión si prevén que parte de la riqueza generada tendrá que ser transferida a los acreedores; y por otra, porque los accionistas tenderán a comportarse de forma oportunista, invirtiendo en proyectos de mayor riesgo que aquellos para los que se recabaron los fondos ajenos.

En Europa y particularmente en España, las empresas presentan estructuras de propiedad más concentradas que las que mantienen las empresas del mundo anglosajón, al tiempo que los intermediarios financieros desarrollan una actuación más activa tanto en la provisión de recursos ajenos como en el gobierno de las empresas. Más aún, el papel que desempeña el mercado como promotor de la eficiencia en la asignación de recursos en estos sistemas financieros es todavía secundarios, pese a haber experimentado un importante desarrollo en los últimos años [Beck et al., 1999; Carlin y Myers, 2003; Beck y Levine, 2004]. En consecuencia, planteamos que la estructura de propiedad es una característica institucional a tener en cuenta en el análisis de las decisiones de endeudamiento de las empresas españolas que son las que en este trabajo centran nuestra atención.

Para alcanzar el objetivo propuesto se parte de los postulados de las denominadas teorías del pecking order [Myers, 1984, y Myers y Majluf, 1984] como la teoría del trade-off [Myers, 1977] aunque introduciendo en el análisis las necesarias modificaciones para considerar la estructura de propiedad de las empresas. Precisamente, uno de los rasgos diferenciales del trabajo radica en el estudio de la relación que se establece entre la estructura de propiedad de las empresas como mecanismo de control de los directivos y el endeudamiento de las mismas [Ergungor, 2004]. En definitiva se trata de contrastar si la elección del nivel de endeudamiento de las empresas españolas que cotizan en bolsa viene determinado por sus oportunidades de crecimiento, por la configuración de su estructura de propiedad y por las características del entorno institucional que las circunda. A este respecto, nuestro trabajo proporciona evidencia empírica adicional sobre la realidad española [Rodríguez, 1997; Cuñat, 1999; Sogorb y López, 2003, y Andrés et al., 2005] y resultados complementarios a los obtenidos en los trabajos empíricos que relacionan las decisiones de endeudamiento con las oportunidades de crecimiento y estructura de propiedad de las empresas del mundo an-

(1) Autores como La Porta et al. [1998 y 2000]; Gallego y Loayza [2000]; Beck y Levine [2002], o Levine [2002] relacionan la estructura de propiedad de las empresas con el sistema financiero del país y su grado de desarrollo económico. 
glosajón [Barclay y Smith, 1995, 1996; Johnson, $1997 a$ y b, 2003; Bevan y Danbolt, 2000; Cantillo y Wright, 2000, y Barclay et al., 2003, entre otros].

El análisis empírico se realiza mediante un modelo de regresión dinámico con datos de panel. La muestra está formada por 111 empresas no financieras que cotizan en el mercado continuo español durante el periodo 1991 a 2001. Con la información disponible construimos un panel de datos incompleto con 912 observaciones. Los estimadores se obtienen tanto con el método en diferencias como con el estimador de sistemas. Afrontamos los problemas de simultaneidad en la estimación mediante la utilización del método de los momentos generalizados [GMM]. Los principales resultados se resumen en lo que sigue: 1) A medida que aumenta la concentración accionarial de las empresas españolas se incrementan los problemas de expropiación de la riqueza no sólo de los accionistas minoritarios sino también de los acreedores, lo cual condiciona negativamente el acceso a las fuentes externas de financiación; 2) Entre las empresas que no generan suficientes fondos internos sólo las que disponen de oportunidades de crecimiento recurren al endeudamiento externo para su financiación; 3) La presencia de accionistas mayoritarios facilita el endeudamiento de aquellas empresas con déficit de financiación, y 4) Las empresas tienden a alcanzar ratios objetivos de apalancamiento a lo largo del tiempo, tal y como se plantea en la teoría del trade-off.

El trabajo se estructura en cinco apartados. Tras esta introducción a la cuestión, el apartado segundo se dedica a revisar los planteamientos teóricos y a la formulación de las hipótesis a contratar. El tercero describe la muestra, variables y metodología empleada en el análisis de los datos. El cuarto apartado prefiere los principales resultados alcanzados tanto a través de los análisis univariantes y multivariantes. Finalmente, en el acápite quinto se presentan las principales conclusiones.

\section{PLANTEAMIENTO TEÓRICO E HIPÓTESIS EMPÍRICAS}

La estructura de capital de las empresas es el resultado de las decisiones financieras adoptadas en el transcurso del tiempo por sus directivos con el fin de crear valor. En este trabajo nos interesamos por las decisiones de endeudamiento de las empresas con oportunidades de crecimiento necesidades externas de financiación dentro del entorno institucional español. Una serie de trabajos, entre los que destacan los de La Porta et al. [1998 y 2000] ${ }^{(2)}$ se han ocupado de forma específica de caracterizar los sistemas financieros de cada país y de evaluar cuán desarrollados se encuentran los mecanismos de protección a sus inversores. Se asume que el entorno institucional determina la toma de decisiones financieras ya que los problemas de agencia y de asimetría de información varían en función del sistema financiero en que opera la empresa [López, 2005]. Entre las manifestaciones del efecto del entorno institucional en la toma de decisiones financieras en la empresa se encuentra tanto la elevada concentración accionarial que se da en algunos países [Burkart et al., 1997; Filatotchev y Mickiewicz, 2002; Gaud et al., 2005, y Jandik y Makhija, 2005], y de forma muy relevante en España, como la fuerte concentración de la propiedad de la deuda, también habitual en España.

(2) Otros trabajos en esta misma línea han sido desarrollados por La Porta et al. [1999]; Johnson y Shleifer [2000]; Allen y Gale [2001]; Levine [2002]; Beck y Levine [2002]; Demirgüç-Kunt y Maksimovic [2002], y Tadesse [2002], entre otros. 
Teniendo en cuenta las características del marco legal e institucional, nuestro estudio trata de analizar los determinantes de las decisiones de endeudamiento de las empresas españolas sobre la base del marco conceptual de las dos principales teorías sobre estructura de capital: la teoría del pecking order y la teoría del trade-off e introduciendo en el análisis la estructura de propiedad como determinante $a$ priori de tales decisiones.

La teoría del pecking order, propuesta por Myers [1984] y Myers y Majluf [1984], considera que los directivos eligen la fuente de financiación que en cada momento esté mejor valorada por el mercado y que en consecuencia, les permita obtener los fondos que necesitan con un menor coste de capital. De entre las alternativas disponibles: fondos propios generados internamente, deuda o ampliación de los capitales propios; la emisión de nuevas acciones es la que plantea mayores problemas de infravaloración por unas de las asimetrías de información. Por su parte los fondos propios generados internamente son los únicos que no se ven afectados por los problemas de asimetría de información asimétrica. Así, el orden que van a seguir los directivos a la hora de financiar sus proyectos de inversión es utilizar primero los fondos propios internos, a continuación endeudarse y sólo en última instanciaemitir nuevas acciones [Chen y Zhao, 2004]. Por tanto, la estructura de capital de cada empresa es el resultado de las decisiones de financiación tomadas a lo largo de los años, decisiones que siguen un orden de preferencias.

La segunda de las teorías propuestas para explicar las decisiones de endeudamiento justifica la existencia de una estructura de capital óptima [Myers, 1977]. Consiguientemente la estructura de capital de las empresas tiende hacia una ratio objetivo que viene determinada por dos efectos de sentido contrario: los costes de quiebra y las ventajas fiscales, que subyacen a los contratos de endeudamiento [Chirinko y Singha, 2000].

Andrés et al. [2000] señalan que las oportunidades rentables de crecimiento a disposición de la empresa o, en su caso, la carencia de ellas, es uno de los principales determinantes del conflicto de agencia entre acreedores y accionistas. De acuerdo con la teoría del pecking order estas oportunidades de crecimiento deberían financiarse siguiendo un orden: primero fondos propios internos, luego deuda y en última instancia emitiendo acciones nuevas. Por tanto, la relación entre deuda y oportunidades de crecimiento dependerá críticamente de que la empresa genera o no suficientes recursos internamente. Por ellos, nuestra primera hipótesis es que aquellas empresas con oportunidades de crecimiento y déficit de fondos generados internamente optarán por la deuda para financiarse.

Nuestra segunda hipótesis es que la financiación de las oportunidades de crecimiento se realizará mediante una combinación de recursos propios y ajenos que permita a la empresa ajustarse a su ratio objetivo [Hackbarth et al., 2004]. En particular, si la empresa tiene elevados niveles de endeudamiento que le acercan peligrosamente a la insolvencia financiera, las oportunidades de crecimiento rentables no deberían financiarse con deuda ya que por un lado los costes de quiebra serán muy elevados, lo que le aleja del ratio objetivo, y por otro lado el problema de subinversión desincentivaría la realización de la inversión [Hovakimian et al., 2004]. El proceso de ajuste al ratio objetivo vendrá pues condicionado por el entorno institucional. Cuanto más eficientes sean los mercados de deuda mas rápido se producirá el ajuste.

Por otro lado, la estructura de propiedad juega un rol importante en los conflictos de agencia tanto entre accionistas y directivos como entre accionistas y acreedores [Himmelberg et al., 1999; Jensen, 2004]. En el caso de las empresas españolas observamos una impor- 
tante concentración accionarial [La Porta et al., 1999], que según señala Jensen [1986] facilita una mejor supervisión de las decisiones de la empresa por los principales accionistas, reduciendo el conflicto de intereses entre internos y externos. La existencia de un accionista o grupo de accionistas con capacidad de control sobre los directivos reduce tanto los problemas de agencia como los de información asimétrica. Por tanto, nuestra tercera hipótesis es que las empresas con accionistas mayoritarios que ejercen funciones de supervisión y control son más proclives al uso de la deuda para financiar sus oportunidades de inversión.

Además, en un contexto como el español en donde la propiedad de las empresas está más concentrada que en entornos como el anglosajón, los problemas de agencia entre accionistas y directivos se disipan a la par que cobran relevancia los problemas entre accionistas mayoritarios y minoritarios. Al ser, pues, diferentes los problemas de agencia parece coherente conjeturar que su influencia sobre las decisiones de endeudamiento será también diferente. La principal preocupación de los mayoritarios será, por un lado, evitar la infravaloración de su propiedad y, por otro, no correr un mayor riesgo no diversificable. Así, nuestra cuarta hipótesis establece que las empresas con propiedad mayoritaria y menor capacidad de autofinanciación optarán por la emisión de deuda para financiar sus proyectos de inversión ya que: evitarán la dilución de la propiedad, evitarán emitir acciones infravaloradas y aprovecharán las ventajas fiscales afrectas al endeudamiento.

\section{MUESTRA, VARIABLES Y METODOLOGÍA}

Para el análisis empírico se ha confeccionado un panel de datos incompleto de las empresas no financieras que cotizan en el mercado continuo español. La razón que justifica la sola utilización de empresas cotizadas es que el análisis de la estructura de propiedad de las empresas sólo tiene sentido cuando la propiedad de las acciones puede cambiar de manos en un mercado líquido, estandarizado y transparente. Además, para el estudio de las oportunidades de crecimiento necesitamos datos sólo disponibles para las empresas cotizadas. Hemos prescindido de todas aquellas observaciones para las que se tenían datos incompletos, al igual que de las empresas no endeudadas, ya que bajo tal escenario no es posible observar los problemas de sustitución de activos y subinversión que en este trabajo se analizan.

La información utilizada para el análisis se obtuvo de la base de datos de la Comisión Nacional del Mercado de Valores [CNMV]. Nuestra muestra incluye 111 empresas con un total de 912 observaciones referidas al período 1991-2001.

Dado que nuestro objetivo es analizar la decisión de endeudamiento, ésta ha sido medida a través de la ratio capitales ajenos sobre activos totales [DTAB]. Entre las variables explicativas se ha considerado, en primer lugar, la concentración de propiedad o capacidad de control y supervisión de los principales accionistas, medida como el porcentaje de propiedad en manos del principal accionista $[\mathrm{C} 1]^{(3)}$. Por su parte, las oportunidades de crecimiento

(3) Como prueba de robustez de los resultados también se han efectuado estimaciones considerando la propiedad accionarial en manos de los dos accionistas mayoritarios [C2] y aquella en manos de los cinco accionistas mayoritarios [C5]. Los resultados obtenidos no se muestran en tablas adjuntas pero pueden ser solicitados directamente a los autores. En su conjunto los resultados se observan como robustos y consistentes para las diversas estimaciones efectuadas. 
se miden con la aproximación de la Q de Tobin $^{(4)}$ [Q]. Las necesidades de fondos externos [DEF] para la financiación de la cartera de proyectos han sido calculadas a través de la variación del activo inmovilizado entre el año actual y el anterior, más la variación del fondo de maniobra entre el año actual y el anterior — que correspondería a las necesidades de fondos para la financiación-, y menos el flujo de tesorería — que se correspondería con los recursos disponibles para la financiación-, y todo escalado por el total de activos [ShyamSunder y Myers, 1999; Sogorb y López, 2003]. Además de las anteriores se han definido tres variables cruzadas que relacionan: la estructura de propiedad de la empresa con la existencia de oportunidades de crecimiento [C1*QI], el déficit de financiación con la estructura de propiedad de la empresa [DEF*C1I] y las oportunidades de crecimiento con el déficit de financiación [Q*DEFI]. La primera de estas variables, C1*QI, toma el valor observado en C1 para todas aquellas empresas que tienen oportunidades de crecimiento y 0 en caso contrario. La segunda variable, DEF*C1I, toma el valor de DEF para todas aquellas empresas en las que el principal accionista posee al menos el 50\% de las acciones, mientras que toma un valor 0 en caso contrario. Por último, la tercera variable cruzada, Q*DEFI, toma el valor de las oportunidades de crecimiento para todas aquellas empresas que presentan déficit de financiación, mientras que toma el valor 0 en caso contrario. Finalmente, incluimos como variables de control el tamaño [LNTAB], la rentabilidad de los activos [ROA], el riesgo de insolvencia [Z], la naturaleza del principal accionista, la relación filial matriz de algunas de las empresas de la muestra (FILIAL) ${ }^{(5)}$ y el sector industrial al que pertenece la empresa. El tamaño de las empresas se mide a partir del valor total de sus activos. En este caso, hemos utilizado su logaritmo neperiano ya que suele ser la solución habitual para trabajar con variables que tienen valores no negativos y de elevada varianza. Para medir la rentabilidad de la cartera de proyectos de las empresas se ha utilizado la ratio de rentabilidad sobre los activos. El riesgo de insolvencia se aproxima a través del valor del Z Score de Altman ${ }^{(6)}$. La naturaleza del principal accionista es una variable cualitativa que considera las siguientes categorías: Banco [BANCO] cuando el principal accionista es un intermediario financiero (banco comercial o caja de ahorro), empresa nacional [EMNAC] si el principal accionista es una empresa de capital nacional, una empresa multinacional [EMMUL] si el principal accionista es una multinacional, empresa pública [EMPUB] si el principal accionista es un organismo o institución pública, o empresa familiar [EMFAM] cuando el principal es una familia. La existencia de empresas cotizadas cuyo principal accionista es otra de las empresas cotizadas se mide con la variable dicotómica FILIAL. Finalmente agrupamos las empresas de la muestra en ocho sectores: Alimentación, pesca y

(4) En la mayoría de los trabajos las oportunidades de crecimiento se miden a través de la aproximación a la $Q$ de Tobin [Johnson, 1997 a y b, 2003; Cuñat, 1999; Krishnaswami et al., 1999; Barclay et al., 2003, Chen y Zhao, 2005, entre otros] y no directamente con la $Q$ de Tobin. La ratio valora mercado de los activos de la empresa sobre el valor en libros de los mismos se aproxima notablemente al valor original de la $Q$. Chung y Pruitt [1994] estiman que al menos el 96,6\% de la variabilidad de la $Q$ de Tobin es explicada por el cociente entre el valor de mercado de los activos y el valor contable de los mismos.

(5) Esta variable nos fue sugerida por uno de los evaluadores anónimos. Nuestro agradecimiento al evaluador por su acertada contribución de la que somos deudores.

(6) El Z Score de Altman es determinado según la siguiente ecuación [Altman, 2002]: Z = 1,2 [fondo de rotación/activo total] $+1,4$ [beneficios retenidos/activo total] $+3,3$ [benéficios antes de intereses e impuestos/activo total] + 0,6 [capitales propios a valor de mercado/pasivos total] $+1,0$ [cifra de negocios/activo total]. También se han efectuado las estimaciones utilizando el Z Score ajustado para aquellas empresas de países desarrollados y para aquellas empresas de países en vías de desarrollo [Altman, 2002]. Si bien los resultados son robustos, hemos decidido conservar aquellos obtenidos con la medida original del Z Score propuesta por Altman [2002]. 
agricultura; cemento y construcción; inmobiliarias; transporte y telecomunicaciones; textil, papel y celulosa; agua, luz, gas y petróleo; servicios; y minería.

Una vez definidas las variables que vamos a utilizar y la muestra de empresas sobre la cual se van a contrastar las hipótesis propuestas, procedemos en dos fases. En la primera fase efectuamos un análisis univariante en el que se describe a la empresa española tipo apoyándonos tanto en la estadística descriptiva como en el análisis de medias. En la segunda fase se realiza un análisis de regresión multivariante aplicando la metodología de datos de panel [Wooldridge, 2002]. Para el análisis de regresión se parte del siguiente modelo estático:

$$
D T A B_{i t}=\beta_{0}+\sum_{j=1}^{n} \beta_{f} X_{j i t}+\varepsilon_{i t}
$$

Donde $j$ representa las distintas variables explicativas, a las empresas que van desde 1 a $111, t$ son los años que van desde 1991 a 2001 y $\varepsilon_{i t}=\eta_{i}+\eta_{t}+e_{i t}$ corresponde al término de error que incluye el efecto individual $\eta_{i}$, el temporal $\eta_{t}$, y el error estocástico $e_{i t}{ }^{(7)}$. Las variables explicativas o independientes son: la estructura de propiedad [C1], las oportunidades de crecimiento [Q], la variable que relaciona la estructura de propiedad de la empresa con la existencia de oportunidades de crecimiento [C1*QI], aquella que relaciona el déficit de financiación con la estructura de propiedad de la empresa [DEF*C1I], aquella que asocia las oportunidades de crecimiento con el déficit de financiación [Q*DEFI], el déficit de financiación [DEF]; la rentabilidad de la cartera de proyectos [ROA], tamaño de la empresa [LNTAB], riesgo de insolvencia [Z], naturaleza del principal accionista [BANCO, EMNAC, EMMUL, EMPUB, EMFAM], relación filial-matriz [FILIAL] y sector de actividad [SECTOR]. También se han incluido variables dummy temporales para cada uno de los años que van desde 1991 a 2001 [DUMMTEMP].

El modelo estático, sin embargo, no es adecuado para contrastar todas nuestras hipótesis por lo que se transforma en un modelo dinámico que permita reflejar el grado de ajuste de la ratio de endeudamiento. En condiciones de mercados perfectos de capitales, la ratio de endeudamiento de la empresa $\left[D T A B_{i t}\right]$ debería ser igual a la ratio de deuda objetivo en el mismo período, es decir, $D T A B_{i t}=D T A B^{*}{ }_{i t}$. Sin embargo, en ausencia de mercados perfectos de capitales existirá un proceso de ajuste en el que $D T A B_{i t}-D T A B_{i t-1}=\lambda_{i t}\left(D T A B_{i t}^{*}-D T A B_{i t-1}\right)$. En esta ecuación $\lambda_{i t}$ corresponde a la velocidad de ajuste. Según la teoría del trade-off este coeficiente de ajuste se aproximaría a $1, \lambda_{i t} \approx 1$. Si dicho coeficiente es igual a 1 , entonces el endeudamiento objetivo es igual a la ratio de endeudamiento observada en el período, alcanzándose las expectativas de forma inmediata y completa [Gujarati, 1990]. Si se observa que $\lambda_{i t}<1$ entonces existe un ajuste parcial entre la ratio de endeudamiento del período $t-1$ al período $t$; sin embargo si $\lambda_{i t}>1$ entonces existe un sobreajuste sobre la ratio objetivo de

(7) El efecto individual, $\eta_{i}$, puede incluir un conjunto de efectos específicos tales como: las barreras de entrada, condiciones generales del mercado de insumos, el riesgo económico de la empresa, entre otros, los cuales se supone que son constantes a lo largo del tiempo. La variable $\eta_{t}$ que representa el efecto temporal incluye los factores macroeconómicos tales como la inflación, las tasas de interés y los shocks de demanda [Sogorb y López, 2003] que afectan en forma transversal al conjunto de todas las empresas en un mismo período. Finalmente, el término de error estocástico $e_{i t}$ captura los posibles errores de medición en las variables independientes, así también como cualquier otra variable independiente que haya sido omitida en el modelo. 
endeudamiento [Soborb y López, 2003]. En consecuencia, la ecuación [1] puede ser reformulada como:

$$
D T A B_{i t}=\lambda_{i t} \cdot \beta_{0}+\left(1-\lambda_{i t}\right) \cdot D T A B_{i t-1}+\lambda_{i t} \cdot\left(\sum_{j=1}^{n} \beta_{j} X_{j i t}\right)+v_{i t}
$$

Téngase en cuenta que el parámetro correspondiente a la variable dependiente con un retardo, $1-\lambda_{i t}$, equivale al coste de ajuste. Además, $v_{i t}=\varepsilon_{i t}-\left(1-\lambda_{i t}\right) \cdot \varepsilon_{i t-1}$; mientras que todos los demás parámetros siguen siendo iguales a los definidos en [1]. En la práctica, una vez obtenida una estimación de $\lambda_{i t}$ a través de la variable dependiente retardada, $Y_{i t-1}$, determinaremos los $\beta_{j}$ dividiendo cada coeficiente del vector $X_{j i t}$ por $\lambda_{i t}$.

Para la estimación de los parámetros del modelo dinámico, ecuación 2, utilizamos la econometría de datos de panel. Consideramos que esta es la metodología adecuada en nuestro caso, dado que disponemos de una muestra de 111 empresas con datos anuales que van desde 1991 a 2001. Entre las ventajas que cita Baltagi [1995] de esta metodología destaca la posibilidad de controlar la heterogeneidad individual de las observaciones —en nuestro caso, cada una de las empresas cotizadas-. Además los datos de panel poseen un mayor contenido informativo, mayor variabilidad, menos colinealidad entre las variables, más grados de libertad y mayor eficiencia. Así mismo, los datos de panel permiten estudiar la dinamicidad de los ajustes y son mejores en la identificación y medición de los efectos que no son detectables a través de los datos de corte transversal o de series temporales. Finalmente, los datos de panel permiten construir y probar modelos conductuales más complejos que aquellos realizados con datos puramente de corte transversal o de serie temporal. En nuestro caso, se utilizan dos métodos de estimación: la estimación en diferencias y el estimador de sistemas.

Los coeficientes del modelo de regresión cuando se dispone de un panel de datos se suelen aproximar con los estimadores within. Sin embargo estos estimadores sólo tienen buenas propiedades cuando se supone que las variables son exógenas, cuestión que no podemos asumir en nuestro trabajo dada la abundante literatura que indica la existencia de endogeneidad cuando se trabaja con variables que miden decisiones financieras. Por ello, se propone utilizar de forma alternativa el método de estimación en primeras diferencias con instrumentos desarrollado por Arellano y Bond [1991] que diferencia las funciones y elimina cualquier sesgo causado por los efectos específicos de las empresas individualmente consideradas a la vez que permite considerar los problemas de endogeneidad. Para ello, estos autores proponen la utilización del Método de Momentos Generalizados [GMM] para instrumentar las variables explicativas a través del uso de valores retardados de los regresores originales, y eliminar así la potencial inconsistencia del parámetro debido a los problemas de endogeneidad. En nuestro caso utilizaremos el método en diferencias GMM en dos etapas con tests estadísticos de robustez para la heteroscedasticidad [Windmeijer, 2000]. Al estudiar la relación entre un conjunto de variables explicativas y la variable dependiente no es posible suponer que los efectos inobservables de las unidades de análisis son igual a cero. El asumir un efecto individual igual a cero - por ejemplo el trabajo de Lang et al. [1996] — no es aplicable en nuestro caso dado que existe una gran heterogeneidad tanto entre las industrias a las que pertenecen las empresas como entre las empresas de una misma industria [Aivazian et al., 2005]. Para controlar este inconveniente de 
la heterogeneidad inobservable individual de la empresa se utilizan las primeras diferencias de las variables originales.

Los estimadores GMM en diferencias [DIF] son superiores a otros métodos de estimación [Antoniou et al., 2002]. Sin embargo el método de estimación en primeras diferencias presenta también algunas desventajas. Blundell y Bond [1998] y Alonso-Borrego y Arellano [1999] han mostrado que para muestras finitas con una pobre precisión asintótica los estimadores en primeras diferencias son débiles y sesgados. También postulan que estos sesgos pueden ser reducidos explotando las restricciones de estacionariedad razonable en el proceso inicial de condiciones. Esto nos conduce a un estimador GMM ampliado en el cual las primeras diferencias retardadas de las series son también usadas como instrumentos por las ecuaciones en niveles [Blundell y Bond, 2000; Blundell et al., 2000; Bond, 2002]. Este estimador es conocido como el estimador de sistemas [SE]. Además, es importante señalar que el estimador de sistemas requiere supuestos más débiles que las condiciones iniciales del resto de los estimadores.

La consistencia del estimador GMM dependerá de la validez del supuesto de que el término de error no muestra una correlación serial de segundo orden y de la validez de los instrumentos. Para tratar estos asuntos, en las regresiones efectuadas se emplean dos pruebas de especificación utilizadas por Arellano y Bond [1991 y 1998]. El primero es el test de Sargan-Hansen de sobreidentificación de restricciones, que se encuentra asintóticamente distribuido como una $X^{2}$. Este test evalúa la validez conjunta de los instrumentos seleccionados. El segundo test corresponde a la hipótesis de que el término de error $\varepsilon_{i t}$ no se encuentra serialmente correlacionado. En este caso, nos centramos en probar que el término de error en diferencias no presenta correlación de segundo orden [por construcción, el término de error en diferencias probablemente presenta correlación de primer orden incluso si el término de error original carece o está exento de ella].

\section{RESULTADOS}

\subsection{Resultados del Análisis Univariante}

A diferencia del modelo financiero anglosajón, la separación de la propiedad y el control es menos apreciable en las empresas españolas que en las empresas habitualmente estudiadas de Estados Unidos o Gran Bretaña. Al reducirse la separación de la propiedad y el control existe un conjunto potencial de problemas de agencia que pueden ser aminorados [Andrés et al., 2000]. Sin embargo, la aparición de estructuras societales concentradas da origen a otro conjunto de conflictos como la elevada concentración del riesgo, la posibilidad de no aprovechar las ventajas de la especialización y el potencial de expropiación de la riqueza de los accionistas minoritarios por parte de los mayoritarios.

En este entorno institucional las empresas no financieras españolas presentan, por término medio, una ratio de deuda sobre el total de activos del $30 \%$ o del $90 \%$ cuando la deuda es escalada sobre el total de capitales propios, así como un predominio de los vencimientos cortos ya que la ratio deuda total a corto plazo sobre deuda total supera el $66 \%$ de promedio [Tabla 1]. 


\section{TABLA 1}

\section{ESTADísTICA DESCRIPTIVA DE LAS VARIABLES}

En esta tabla se incluye la media, valores mínimo y máximo, desviación típica y varianza, de las siguientes variables: deuda total sobre activos totales [DTAB], deuda total sobre recursos propios [DTEB], deuda total de largo [DTLDT] y de corto [DTCDT] plazo sobre deuda total, propiedad en manos del principal accionista [C1], valor de mercado de los activos sobre el valor contable de los mismos [Q], déficit de fondos para la financiación de las variaciones de activos fijos y el fondo de maniobra [DEF], logaritmo neperiano del total de activos contables en miles de euros [LNTAB], Z es el coeficiente Z Score de Altman y el beneficio neto sobre el total de activos [ROA]. También se han incluido las variables que representan la naturaleza del inversor principal: empresa familiar [EMFAM], sociedad de inversión [SOCINV], empresa nacional [EMNAC], empresa multinacional [EMMUL] y empresa pública [EMPUB].

\begin{tabular}{|lcc|c|c|c|}
\hline \multicolumn{1}{|c|}{ VARIABLES } & MEDIA & MinImo & MáxImo & DESV. TíPICA & VARIANZA \\
\hline DTAB & 0.3004 & 0.0002 & 0.8717 & 0.1845 & 0.0340 \\
\hline DTEB & 0.9025 & 0.0003 & 62.2390 & 2.4542 & 6.0233 \\
\hline DTLDT & 0.3395 & 0.0000 & 1.0000 & 0.3069 & 0.0942 \\
\hline DTCDT & 0.6605 & 0.0000 & 1.0000 & 0.3069 & 0.0942 \\
\hline C1 & 40.2273 & 0.0110 & 99.2000 & 26.3289 & 693.2086 \\
\hline$Q$ & 1.1864 & 0.2275 & 30.5235 & 1.1906 & 1.4175 \\
\hline DEF & -0.0611 & -1.6254 & 0.8526 & 0.1976 & 0.0391 \\
\hline LNTAB & 10.7887 & 7.2779 & 17.7805 & 1.7461 & 3.0489 \\
\hline Z & 2.7282 & -3.6421 & 135.6019 & 6.9936 & 48.9106 \\
\hline ROA & 0.0260 & -0.8190 & 0.2240 & 0.0800 & 0.0064 \\
\hline EMFAM & 0.2588 & 0.0000 & 1.0000 & 0.4382 & 0.1920 \\
\hline SOCINV & 0.1919 & 0.0000 & 1.0000 & 0.3940 & 0.1552 \\
\hline EMNAC & 0.2555 & 0.0000 & 1.0000 & 0.4364 & 0.1904 \\
\hline EMMUL & 0.1853 & 0.0000 & 1.0000 & 0.3888 & 0.1511 \\
\hline EMPUB & 0.1086 & 0.0000 & 1.0000 & 0.3112 & 0.0969 \\
\hline ObS. & 912 & & & \\
\hline
\end{tabular}

La concentración accionarial sigue siendo una de las principales características de las empresas españolas cotizadas. Así, en la Tabla 1 observamos que por término medio el accionista mayoritario $^{(8)}$ llega a poseer el $40 \%$ de las acciones, porcentaje significativamente mayor del que se observa en los entornos financieros basados en la ley común [La Porta et al., 1998]. Además, las empresas españolas cuentan con oportunidades de crecimiento [valor de la Q superior a 1] y una razonable salud financiera [el Z Score de Altman alcanza una media de 2,73].

El análisis descriptivo se completa con un análisis de medias. En este caso la muestra se divide en tres submuestras de igual tamaño en función de la variable deuda total sobre el total de activos [DTAB] y deuda total sobre capitales propios [DTEB]. Cada una de las submuestras contiene aquellas observaciones con los valores más altos, intermedios y más bajos de DTAB y DTEB. Para cada submuestra se calculan los valores promedios de las variables objeto de estudio. Dado nuestro objetivo de investigación, para resaltar las diferencias comparamos las

(8) No siempre el porcentaje de acciones en manos del principal accionista es una adecuada medida del grado de concentración de la propiedad en una empresa. Por esta razón los análisis realizados en el trabajo considerando el principal accionista se han repetido utilizando como medidas alternativas de concentración de la propiedad la suma de las participaciones de los dos accionistas mayoritarios o de los cinco accionistas mayoritarios. Además, la elevada participación del accionista mayoritario en el capital de las empresas que se observa en España, facilita que podamos utilizar esta medida como aproximación de la existencia de accionistas con capacidad de control y supervisión sobre las decisiones de la empresa. 
medias de las dos submuestras que contienen los valores extremos, prescindiendo entonces de los resultados obtenidos con la submuestra central. Los resultados de este análisis de medias [Tabla 2] nos indican que las empresas con mayor nivel de deuda, medida tanto sobre el total de activos como sobre los capitales propios, poseen a su vez una menor participación del accionista mayoritario y menores oportunidades de crecimiento que aquellas empresas con menor deuda registrada en sus libros. Por otra parte, las empresas con elevados volúmenes de deuda [DTAB y DTEB] poseen también menores niveles de déficit de fondos para la financiación de sus carteras de proyectos de inversión. En relación a nuestras variables de control, toda vez que mayores sean los niveles de deuda, también se observarán mayores tamaños empresariales, mayor riesgo de quiebra y menores niveles de rentabilidad de los activos.

\section{TABLA 2}

\section{ANÁLISIS DE DIFERENCIA DE MEDIA DE LAS VARIABLES}

La tabla muestra las pruebas de diferencias de medias para las variables deuda total sobre activos totales [DTAB] y deuda total sobre capitales propios [DTEB]. Para llevar a cabo el análisis se han clasificado las observaciones en tres tercios, considerándose solamente los tercios superior e inferior. La tabla muestra además la media alcanzada por el conjunto de variables para el tercio inferior [1] y para el tercio superior [3]. La prueba de diferencia de medias para cada tercio y la significación estadística nos permite probar la hipótesis nula de que no existen diferencias entre los valores promedio.

\begin{tabular}{|c|c|c|c|c|c|}
\hline & & \multicolumn{2}{|c|}{$D T A B$} & \multicolumn{2}{|c|}{ DTEB } \\
\hline Variables & Tercio & Medias & $\begin{array}{l}\text { Dif. Med. } \\
\text { [Sig. Bil.] }\end{array}$ & Medias & $\begin{array}{l}\text { Dif. Med. } \\
\text { [Sig. Bil.] }\end{array}$ \\
\hline DTAB & $\begin{array}{l}1 \\
3\end{array}$ & $\begin{array}{l}0,0954 \\
0,5129\end{array}$ & $\begin{array}{c}-0,4175 \\
0,0000 \\
\end{array}$ & $\begin{array}{l}0,1002 \\
0,5080\end{array}$ & $\begin{array}{c}-0,4078 \\
0,0000\end{array}$ \\
\hline DTEB & $\begin{array}{l}1 \\
3 \\
\end{array}$ & $\begin{array}{l}0,1583 \\
1,9412\end{array}$ & $\begin{array}{c}-1,7829 \\
0,0000 \\
\end{array}$ & $\begin{array}{l}0,1466 \\
1,9996\end{array}$ & $\begin{array}{c}-1,8530 \\
0,0000 \\
\end{array}$ \\
\hline DTLDT & $\begin{array}{l}1 \\
3 \\
\end{array}$ & $\begin{array}{l}0,2563 \\
0,4002 \\
\end{array}$ & $\begin{array}{c}-0,1440 \\
0,0000 \\
\end{array}$ & $\begin{array}{l}0,2447 \\
0,3991 \\
\end{array}$ & $\begin{array}{c}-0,1545 \\
0,0000 \\
\end{array}$ \\
\hline DTCDT & $\begin{array}{l}1 \\
3 \\
\end{array}$ & $\begin{array}{l}0,7437 \\
0,5998 \\
\end{array}$ & $\begin{array}{l}0,1440 \\
0,0000\end{array}$ & $\begin{array}{l}0,7553 \\
0,6009 \\
\end{array}$ & $\begin{array}{l}0,1545 \\
0,0000\end{array}$ \\
\hline PA1 & $\begin{array}{l}1 \\
3 \\
\end{array}$ & $\begin{array}{l}41,0943 \\
38,9006 \\
\end{array}$ & $\begin{array}{l}2,1937 \\
0,3067\end{array}$ & $\begin{array}{l}41,6836 \\
38,3006 \\
\end{array}$ & $\begin{array}{l}3,3830 \\
0,1140 \\
\end{array}$ \\
\hline$Q$ & $\begin{array}{l}1 \\
3 \\
\end{array}$ & $\begin{array}{l}1,3688 \\
1,1038 \\
\end{array}$ & $\begin{array}{l}0,2651 \\
0,0207\end{array}$ & $\begin{array}{l}1,3578 \\
1,1073 \\
\end{array}$ & $\begin{array}{l}0,2504 \\
0,0293 \\
\end{array}$ \\
\hline$D E F$ & $\begin{array}{l}1 \\
3 \\
\end{array}$ & $\begin{array}{l}-0,0497 \\
-0,0570 \\
\end{array}$ & $\begin{array}{l}0,0073 \\
0,6578 \\
\end{array}$ & $\begin{array}{l}-0,0480 \\
-0,0659 \\
\end{array}$ & $\begin{array}{l}0,0179 \\
0,2637 \\
\end{array}$ \\
\hline$\angle N T A B$ & $\begin{array}{l}1 \\
3 \\
\end{array}$ & $\begin{array}{l}10,5614 \\
11,2804 \\
\end{array}$ & $\begin{array}{r}-0,7191 \\
0,0000 \\
\end{array}$ & $\begin{array}{l}10,4385 \\
11,2092 \\
\end{array}$ & $\begin{array}{c}-0,7707 \\
0,0000 \\
\end{array}$ \\
\hline$Z$ & $\begin{array}{l}1 \\
3\end{array}$ & $\begin{array}{l}4,9663 \\
1,2726\end{array}$ & $\begin{array}{l}3,6937 \\
0,0000\end{array}$ & $\begin{array}{l}5,0206 \\
1,2587\end{array}$ & $\begin{array}{l}3,7618 \\
0,0000\end{array}$ \\
\hline$R O A$ & $\begin{array}{l}1 \\
3 \\
\end{array}$ & $\begin{array}{r}0,0559 \\
-0,0021 \\
\end{array}$ & $\begin{array}{l}0,0581 \\
0,0000\end{array}$ & $\begin{array}{r}0,0569 \\
-0,0057 \\
\end{array}$ & $\begin{array}{l}0,0626 \\
0,0000\end{array}$ \\
\hline EMFAM & $\begin{array}{l}1 \\
3 \\
\end{array}$ & $\begin{array}{l}0,2303 \\
0,2039 \\
\end{array}$ & $\begin{array}{l}0,0263 \\
0,4321\end{array}$ & $\begin{array}{l}0,2467 \\
0,2105 \\
\end{array}$ & $\begin{array}{l}0,0362 \\
0,2889 \\
\end{array}$ \\
\hline SOCINV & $\begin{array}{l}1 \\
3 \\
\end{array}$ & $\begin{array}{l}0,1908 \\
0,2533 \\
\end{array}$ & $\begin{array}{c}-0,0625 \\
0,0639 \\
\end{array}$ & $\begin{array}{l}0,1776 \\
0,2336 \\
\end{array}$ & $\begin{array}{c}-0,0559 \\
0,0883 \\
\end{array}$ \\
\hline EMNAC & $\begin{array}{l}1 \\
3 \\
\end{array}$ & $\begin{array}{l}0,2895 \\
0,2237\end{array}$ & $\begin{array}{l}0,0658 \\
0,0635 \\
\end{array}$ & $\begin{array}{l}0,2796 \\
0,2467 \\
\end{array}$ & $\begin{array}{l}0,0329 \\
0,3579 \\
\end{array}$ \\
\hline EMMUL & $\begin{array}{l}1 \\
3 \\
\end{array}$ & $\begin{array}{l}0,2007 \\
0,2007\end{array}$ & $\begin{array}{l}0,0000 \\
1,0000 \\
\end{array}$ & $\begin{array}{l}0,2237 \\
0,1941 \\
\end{array}$ & $\begin{array}{l}0,0296 \\
0,3701 \\
\end{array}$ \\
\hline EMPUB & $\begin{array}{l}1 \\
3\end{array}$ & $\begin{array}{l}0,0888 \\
0,1184\end{array}$ & $\begin{array}{c}-0,0296 \\
0,2317\end{array}$ & $\begin{array}{l}0,0724 \\
0,1151\end{array}$ & $\begin{array}{c}-0,0428 \\
0,0707\end{array}$ \\
\hline Obs. & & 304 & & 304 & \\
\hline
\end{tabular}


En consecuencia, los resultados del análisis de medias constituyen indicios de que aquellas empresas capaces de generar mayores flujos de tesorería serán las menos endeudadas. Aparentemente, estos resultados confirmarían los postulados de la teoría del pecking order. Las empresas españolas tienden a utilizar en primer lugar los recursos generados internamente, y posteriormente cuando éstos ya hayan sido agotados, estarán dispuestos a soportar mayores ratios de apalancamiento.

\subsection{Resultados del Análisis Multivariante}

Las estimaciones se han efectuado con los dos métodos antes comentados: el estimador de sistemas [SE] y las estimaciones en diferencias [DIF]. El test de Wald ha sido utilizado para probar si tanto el modelo en su conjunto, Ecuación 2, como las variables dummy utilizadas son estadísticamente significativos ${ }^{(9)}$. Además se empleó el test de Sargan-Hansen de sobreidentificación de restricciones que permite aceptar la hipótesis nula de que el modelo está bien identificado. Todas las especificaciones del modelo son estadísticamente significativas, los instrumentos están bien especificados y no existe correlación serial de segundo orden en ningún caso. Los estimadores obtenidos se presentan en la Tabla 3.

\section{TABLA 3}

\section{DETERMINANTES DEL ENDEUDAMIENTO DE LAS EMPRESAS ESPAÑOLAS}

La tabla contiene los resultados de las regresiones realizadas sobre una muestra de 111 empresas españolas no financieras en el periodo 1991-2001. La regresión estimada corresponde a la ecuación [2] explicitada en el apartado de metodología. La variable dependiente es DTAB, que corresponde a la deuda total sobre activos totales. Las variables independientes son: $\mathrm{C} 1$ que corresponde al porcentaje de propiedad en manos del principal accionista, $\mathrm{C} 1^{\star} \mathrm{Q}$ que corresponde a la relación entre la concentración de propiedad y las oportunidades de crecimiento, $Q$ a las oportunidades de crecimiento medidas como la ratio valor mercado a valor libro, $Q^{\star} D E F I$ que corresponde a la relación entre las oportunidades de crecimiento, el déficit de fondos para la financiación de la cartera de proyectos, DEF, $\mathrm{DEF}^{\star} \mathrm{C} 1 \mathrm{l}$ que corresponde a la relación entre el déficit de fondos y la concentración de propiedad, $\mathrm{DTAB}_{\mathrm{t}-1}$ como el retardo de la variable dependiente, el tamaño LNTAB es aproximado a través del logaritmo neperiano del total activos, el riesgo de insolvencia Z que equivale al Z Score de Altman y ROA que relaciona el beneficio neto con el total activo. Se introducen las variables dummy correspondientes a la naturaleza del principal inversor. En todos los casos el test de Wald en sus diversas aplicaciones indica que los modelos son estadísticamente significativos. Las variables $C 1, Q, C 1{ }^{\star} \mathrm{QI}, \mathrm{DEF}{ }^{\star} \mathrm{C} 1 \mathrm{l}$ y $\mathrm{Q}{ }^{\star} \mathrm{DEFI}$ se han considerado endógenas y han sido convenientemente instrumentadas a través de GMM. El test de Sargan-Hansen indica que el modelo está bien especificado. No se observa la existencia de autocorrelación de segundo orden en ninguno de los modelos. Significación estadística: *** al nivel del $1 \%,{ }^{* *}$ al $5 \%$ y ${ }^{*}$ al $10 \%$, registrada bajo cada coeficiente.

\begin{tabular}{|c|c|c|c|}
\hline SISTEMA DE ESTIMACIÓN & $S E$ & DIF & $S E$ \\
\hline Variables & $\begin{array}{c}\text { Coeficiente } \\
\text { Error Estándar }\end{array}$ & $\begin{array}{c}\text { Coeficiente } \\
\text { Error Estándar }\end{array}$ & $\begin{array}{l}\text { Coeficiente } \\
\text { Error Estándar }\end{array}$ \\
\hline CONST & $\begin{array}{l}0,1035 \quad * * * \\
0,0084\end{array}$ & & $\begin{array}{l}0,1537^{\text {***}} \\
0,0144\end{array}$ \\
\hline $\mathrm{C} 1$ & $\begin{array}{r}-0,0014 \quad * * * \\
0,0001\end{array}$ & $\begin{array}{l}-0,0007 \text { *** } \\
0,0000\end{array}$ & $\begin{array}{l}-0,0013 \text { *** } \\
0,0001\end{array}$ \\
\hline $\mathrm{C} 1^{*} \mathrm{QI}$ & $\begin{array}{l}0,0008 \quad * * * \\
0,0000\end{array}$ & $\begin{array}{l}0,0006^{* * *} \\
0,0000\end{array}$ & $\begin{array}{l}0,0008 * * * \\
0,0000\end{array}$ \\
\hline$C 1+C 1^{*} Q$ & $-0,0007$ & $-0,0001$ & $-0,0005$ \\
\hline
\end{tabular}

(Continúa pág. sig.)

(9) Se han realizado tests de Wald para comprobar: i) La significación conjunta de los parámetros, ii) La significación de las variables dummy temporales, y iii) La significación de las variables de sector industrial. 
TABLA 3

Determinantes del endeudamiento de las empresas ESPaÑolas (CoNT.)

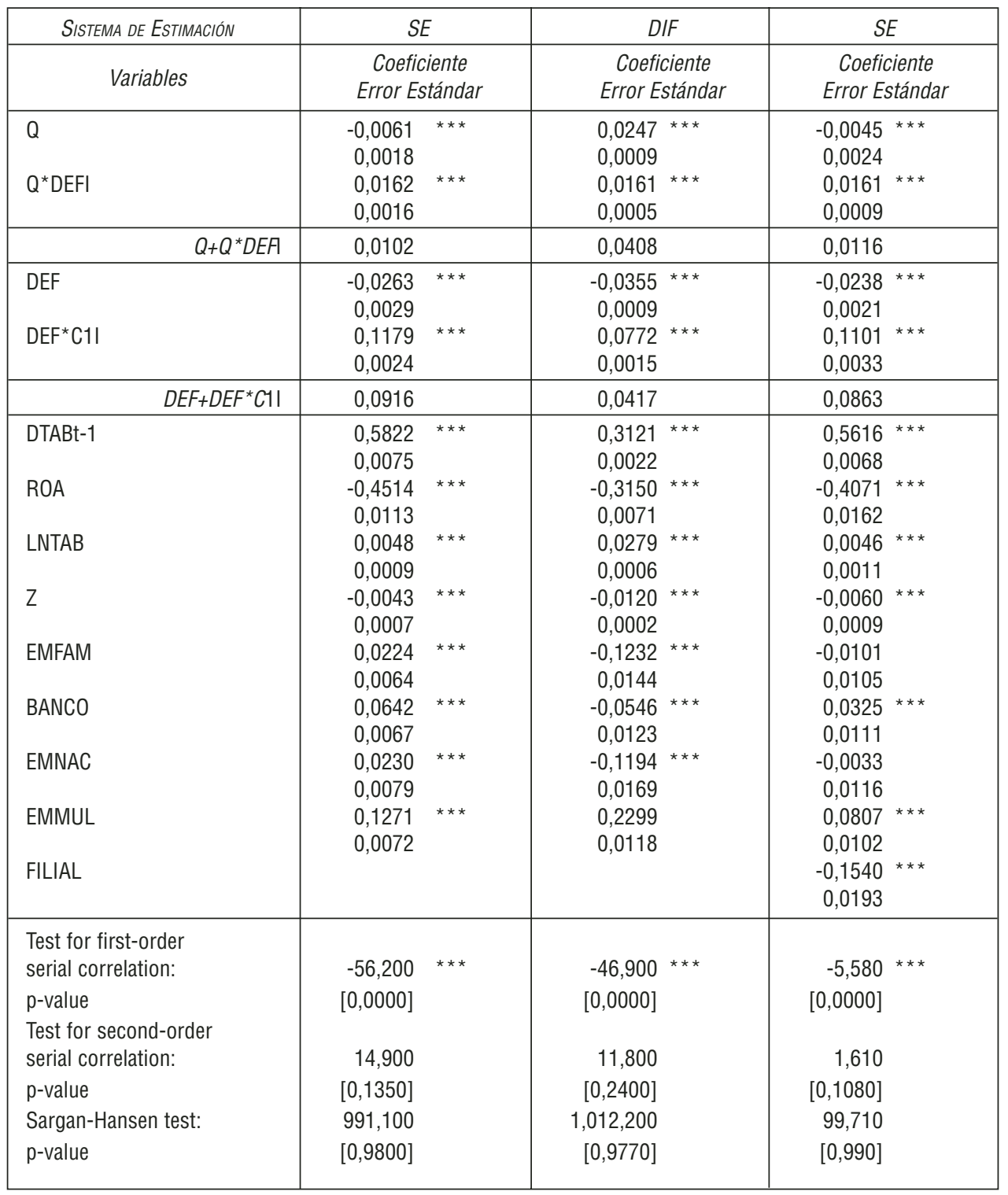

En primer lugar, observamos que la relación entre las oportunidades de crecimiento y el nivel de deuda, si bien es estadísticamente significativa, es distinta dependiendo del método de estimación empleado [en diferencias o en el estimador de sistemas]. Tal disparidad impide avanzar una conclusión clara al respecto, al tiempo que apunta a la necesidad de utilizar una medida mas ajustada a nuestro objetivo de contrastar si las empresas utilizan deuda para financiar sus oportunidades de crecimiento una vez agotados los recursos generados internamente. Por esta razón se utiliza la variable cruzada Q*DEFI que mide las 
oportunidades de crecimiento en empresas que han agotado su capacidad de autofinanciación. Los resultados nos permiten verificar nuestra primera hipótesis. Con respecto a la misma, observamos que las empresas españolas con oportunidades de crecimiento incrementan su nivel de deuda una vez que han agotado sus recursos generados internamente. Estos resultados confirman las propuestas de la teoría del pecking order.

También se confirma nuestra segunda hipótesis de que las empresas españolas tratan de alcanzar una ratio objetivo de endeudamiento. Así, observamos que la variable dependiente retardada un período $\left[\mathrm{DTAB}_{\mathrm{t}-1}\right]$ registra una relación positiva y estadísticamente significativa. Sin embargo, la velocidad de ajuste hacia el nivel de endeudamiento óptimo difiere en función del método de estimación utilizado. Así mismo, nuestros resultados difieren de los obtenidos por Sogorb y López [2003] para un conjunto de medianas empresas españolas no cotizadas. Según estos autores el coeficiente de ajuste para su muestra de empresas es 0,862 mientras que en nuestro caso toma un valor igual a 0,688 [=1-0,3121] si realizamos la estimación por el método en diferencias y es igual a 0,418 [=1-0,5822] cuando la estimación se realiza con el estimador de sistemas. En definitiva obtenemos que existe una ratio objetivo de endeudamiento que las empresas españolas tratan de alcanzar con sus decisiones de endeudamiento y que la velocidad del ajuste a dicho ratio es distinta en nuestra muestra de empresas cotizadas a la estimada por Sogorb y López [2003] para las no cotizadas.

El papel destacado que juegan los intermediarios financieros bancarios en el sistema financiero español hace que gran parte de la deuda de las empresas sea de naturaleza bancaria. El endeudamiento bancario tiene ventajas frente a la emisión de deuda en los mercados en lo que atañe a flexibilidad en las cláusulas contractuales y renegociación de las mismas entre las partes. Por lo tanto, para las empresas españolas sería posible alcanzar su ratio objetivo de endeudamiento con plazos y costes de ajustes menores que los de las empresas que operan en entornos basados en common-lawr que suelen mantener en sus balances una menor proporción de financiación intermediaria.

Los resultados muestran también una relación negativa y estadísticamente significativa entre la concentración de la propiedad en manos del principal accionista y la ratio de endeudamiento. Esta relación confirma que a medida que aumenta la capacidad de control por parte de los accionistas mayoritarios se reduce el endeudamiento bien sea para evitar los problemas de subinversión y sustitución de activos bien porque el accionista mayoritario desea prescindir del control externo adicional que comporta el recurso al endeudamiento. Una elevada concentración accionarial puede ser un mecanismo generador de valor para la empresa al reducir los costes de agencia pero ese mecanismo tiene un límite a partir del cual se destruye valor y se agudizan los problemas de agencia [McConnell y Servaes, 1990, 1995; Azofra et al., 1995; Maug, 1998, y Andrés et al., 2000]. Estos problemas se traducen básicamente en el de sustitución de activos, pues al fomentar un alineamiento mayor de intereses entre accionistas y directivos, los primeros pueden incentivar a los segundos a realizar proyectos de inversión de mayor riesgo. Por otro lado, cuando el gasto de concentración de la propiedad supera el nivel óptimo se acentúa el riesgo de expropiación no sólo de la riqueza no sólo de los demás accionistas, sino también, como en el caso del problema de sustitución de activos, de la riqueza de los acreedores. Consiguientemente a medida que aumenta la participación accionarial del principal accionista se reducen las probabilidades de captar financiación externa en condiciones económicamente aceptables. Sin embargo esta relación negativa 
se modera cuando la empresa dispone de oportunidades de crecimiento. De hecho, se valida nuestra tercera hipótesis de que las empresas de propiedad concentrada en el principal accionistas recurren en mayor medida al endeudamiento cuando tienen oportunidades de conocimiento que cuando carecen de ellas... aparentemente por la existencia de oportunidades de crecimiento incentiva el recurso a la financiación ajena. Pero también la elevada concentración accionarial característica de las empresas españolas incrementa el riesgo potencial de expropiación de la riqueza, tanto de los accionistas minoritarios como de los acreedores, lo que a su vez limita su acceso a las fuentes externas de financiación.

Constatamos, además, que la capacidad de la empresa para generar fondos internamente con los que financiar su cartera de proyectos parece influir sobre su nivel de endeudamiento. Nuestros hallazgos apoyan la hipótesis de que las empresas con mayores necesidades de financiación externa, DEF, son las que tienen menor capacidad para captar fondos debido a que en ellas se acentúa el problema de selección adversa. Sin embargo, tal relación se modifica cuando la empresa tiene control mayoritario. En cuyo caso observamos que cuando la empresa necesita financiación externa [DEF+DEF*C1I] incrementa su endeudamiento, lo que es congruente con nuestra cuarta hipótesis según la cual el accionista mayoritario de una empresa con necesidades de financiación externa preferirá aumentar el endeudamiento a financiarse emitiendo nuevas acciones, tanto para evitar la infravaloración de sus acciones, como para eludir un incremento del riesgo específico de la empresa. Estos resultados proporcionan, siquiera parcialmente, soporte empírico a la teoría del pecking order ya que el recurso a la financiación externa se produce cuando se han agotado los fondos generados internamente, y además se prefiere la deuda a la emisión de nuevas acciones.

Se evidencia, además, que la ratio de endeudamiento de una empresa viene determinada por su tamaño [LNTAB], por su probabilidad de quiebra y por su rentabilidad. Los resultados muestran una relación directa entre el tamaño y el nivel de deuda de las empresas y una relación negativa entre ROA y endeudamiento y entre Z y endeudamiento. El tamaño está relacionado con las garantías colaterales que puede ofrecer la empresa en el momento de suscribir los contratos de deuda. De este modo, cuanto mayor es el volumen de activos empresariales, mayor es la capacidad de ofrecer garantías colaterales y por consiguiente, menores son las barreras que debe sortear la empresa para la emisión de deuda. Por su parte, las empresas más rentables dispondrán de recursos internos para su financiación por lo que demandarán una menor cantidad de fondos externos. E igualmente observamos que las empresas con una menor probabilidad de insolvencia - mayor Z scoreson las menos endeudadas. Finalmente, observamos que las empresas cuyo principal accionista es otra de las empresas de la muestra (FILIAL) se endeudan en menor medida ya que pueden utilizar la capacidad de endeudamiento del grupo en su conjunto.

\section{CONCLUSIONES}

El objetivo de este trabajo ha sido analizar los determinantes del endeudamiento de las empresas españolas durante la década de los noventa. Para ello proporcionamos un modelo que parte de los planteamientos teóricos existentes para relacionar las decisiones de endeudamiento con el conjunto de oportunidades de crecimiento, la capacidad de autofinanciación de las empresas, su tamaño, su rentabilidad y su probabilidad de quiebra, y que ha sido convenientemente modificado para dar cabida a las características de la estructura de 
propiedad de los capitales propios de las empresas españolas. Estructura de propiedad que difiere entre países y que consideramos influye en la toma de las decisiones financieras.

El modelo se estima tanto con el método en diferencias como con el estimador de sistemas propuestos por Arellano y Bond [1991] y Alonso-Borrego y Arellano [1999], respectivamente; con el objeto de poder contrastar nuestras hipótesis y probar la robustez de los resultados alcanzados.

Los resultados alcanzados nos permiten concluir que, en primer lugar y al hilo del planteamiento de nuestras hipótesis, el sistema financiero continental y el marco legal e institucional en el que se inscribe la actividad de las empresas españolas — civil-law-, encuentran en la concentración accionarial de los capitales propios una solución a los problemas de agencia. Y es este mecanismo de gobierno corporativo el que permite a las empresas con oportunidades de crecimiento acceder con mayor facilidad a los mercados de deuda. En este sentido, observamos que las empresas españolas utilizan el endeudamiento para financiar sus oportunidades de crecimiento una vez que han agotado sus recursos internos y, en particular, aquellas empresas con una estructura de propiedad más concentrada. Esta relación directa es contraria a la observada en los países cuyo sistema financiero desciende de la rama anglosajona —common-law — [Johnson, 1997 a; Houston y James, 1996]. En el caso de las empresas españolas, el elevado porcentaje de acciones en manos del principal accionista (incluso de los cinco principales accionistas) permite alinear los intereses de las partes y minimizar, por ello, los potenciales costes de agencia que dichos problemas implican.

Como segunda constatación, y a su vez derivada de la anterior, también hemos observado que aquellas empresas caracterizadas tanto por tener déficit de financiación como por poseer una estructura de propiedad dominada por un accionista principal (los resultados se mantienen para el caso de cinco accionistas), tienden también a incrementar su ratio de apalancamiento. Este resultado es congruente con la hipótesis de que el accionista con propiedad mayoritaria intentará evitar tanto la infravaloración de sus acciones como la dilución del capital a través de la emisión de deuda y no de nuevas acciones o el incremento del riesgo específico en la empresa.

Una tercera conclusión es que las empresas españolas tienden hacia una ratio de endeudamiento óptima. La velocidad de ajuste hacia tal ratio objetivo difiere de la obtenida para muestras de empresas del ámbito anglosajón e incluso difiere de la obtenida para una muestra de empresas españolas no cotizadas.

Se observa además que la ratio de endeudamiento de las empresas viene determinada por su tamaño, por su probabilidad de quiebra, y por su rentabilidad. A mayor tamaño empresarial mayor es el nivel de endeudamiento al que pueden acceder las empresas debido a los mayores garantías colaterales que pueden ofrecer. Por otra parte, las empresas con mayores ratios de apalancamiento son las que tienen una mayor probabilidad de quiebra.

Finalmente, aunque nuestros resultados no son concluyentes ponen de manifiesto que las decisiones de endeudamiento de las empresas españolas no se enmarcan claramente en ninguna de las dos teorías sobre estructura de capital consideradas. Así entre los resultados que pudieran indicar que las empresas españolas tienden a establecer una jerarquía en sus decisiones de endeudamiento, destaca el que a medida que se incrementan las oportunidades de crecimiento aumenta también el apalancamiento. No obstante, nuestros re- 
sultados también revelan que las empresas con mayor necesidades de financiación poseen menor capacidad para captar fondos externamente debido al incremento del riesgo de incurrir en problemas de selección adversa. Sin embargo, si en dichas empresas existen oportunidades rentables de crecimiento, éstas recurrirán, una vez agotados los recursos internos, al endeudamiento para financiar sus oportunidades de crecimiento. Así mismo, la relación negativa entre rentabilidad y endeudamiento avala la teoría del pecking order. Por otro lado, la decisión de endeudamiento también podría responder a una ratio objetivo de endeudamiento que vendría determinada, por las características del sistema financiero español, el cual permitiría un ajuste más dinámico hacia esta ratio.

\section{REFERENCIAS BIBLIOGRÁFICAS}

Allen, F., y GALE, D. [2001]: Comparing financial systems, 1. ${ }^{a}$ Edición, MIT Press, Paperback Edition, Massachusetts].

Alonso-Borrego, C., y Arel.ANo, M. [1999]: «Symmetrically normalized instrumental variable estimation using panel data», Journal of Business \& Economic Statistics, 17, pp. 36-49.

ALtмan, E. [2002]: Bankruptcy, credit risk, and high yield bonds, Blackwell Publishers.

Andrés; Pablo; López, F.; Rodríguez, J. A., y Vallelado, E. [2005]: «Determinants of bank debt in a continental financial system: evidence from Spanish companies», Financial Review, 40, 3, pp. 305-334.

Andrés; Pablo; Azofra, V., y RodríGuez, J. A. [2000]: «Endeudamiento, oportunidades de crecimiento y estructura contractual: un contraste empírico para el caso español»; Investigaciones Económicas, 24, 3, pp. 641-679.

Areldano, M., y Bond, S. [1991]: «Some tests of specification for panel data: Monte Carlo evidence and an application to employment equation», Review of Economic Studies, 58, pp. 277-297.

— [1998]: «Dynamic panel data estimation using DPD98 for Gauss», A guide for users, pp. 1-27.

Areldano, M., y Bover, O. [1990]: «La econometría de datos de panel», Investigaciones Económicas (Segunda Época) 14, 1, pp. 3-45.

Azofra, V.; Rodríguez, J. A., y VALLeLAdo, E. [1995]: «Estructura de propiedad, endeudamiento y resultados en la gran empresa industrial española: un enfoque de agencia», Actas de las X Jornadas de Economía Industrial, pp. 129-145.

BALTAGI, B. [1995]: Econometric analysis of panel data, Ed. John Wiley \& Sons Ltd., (Baffins Lane, Chichester, Inglaterra).

BARCLAY, M., y SмITH, C. [1995]: «The maturity structure of corporate debt», The Journal of Finance, 50, 2, pp. 609-631.

BARClAY, M., y SмITH, C. [1996]: «On financial architecture: leverage, maturity and priority», Journal of Applied Corporate Finance, 8, 4, pp. 4-17.

BarCLAY, M.; Marx, L., y Sмith, C. [2003]: «The joint determination of leverage and maturity», Journal of Corporate Finance, 9, pp. 149-167.

Thorsten, B., y Ross Levine [2002]: «Industry growth and capital allocation: does having a market or bank-based system matter?», National Bureau of Economic Research, Working Paper, 8982, pp. 1-35.

Thorsten, B., y Levine, R. [2004]: «Stock market, banks, and growth: Panel evidence», Journal of Banking \& Finance, 28, pp. 423-442.

Thorsten, B.; Demirgüç-Kunt, A., y Levine, R. [1999]: «A new database on financial development and structure», Financial Sector Discussion Paper, n. ${ }^{\circ}$ 2, The World Bank, pp. 1-26.

Bevan, A., y Jo Danbolt [2000]: «Capital structure and its determinants in the United Kingdom: a compositional analysis», Working Paper Series 2000/2, University of Glasgow, pp. 1-30. 
BRAILSFORD, Timothy y DANIEL Yeoh [2004]: «Agency problems and capital expenditure announcements», Journal of Business, 77, 2, pp. 223-256.

Burkart, M., Gromb, D., y PANunzi, F. [1997]: «Large shareholders, monitoring, and the value of the firm», Quarterly Journal of Economics, 112, 3, pp. 693-728.

Cantillo, M. y Wright, J. [2000]: «How do firms choose their lenders? An empirical investigation», Review of Financial Studies, 13, 1, pp. 155-189.

CARLIN, W., y MAYER, C. [2003]: «Finance, investment, and growth», Journal of Financial Economics, 69, pp. 191-226.

ChEN, Long, y XinLeI, Z. [2004]: «The modified pecking order theory: New evidence from corporate financing decisions», Artículo no publicado, pp. 1-43.

- [2005]: «0n the relation between market-to-book ratio, growth opportunity, and leverage ratio», Artículo no publicado, pp. 1-43.

CHIRINKo, R., y SingHA, A. [2000]: «Testing static tradeoff against pecking order models of capital structure: A critical comment», Journal of Financial Economics, 58, pp. 417-425.

Chung; Kee, H., y Pruttr, S. W. [1994]: «A simple approximation of Tobin's Q», Financial Management, 23, pp. 70-74.

Cuñat, V. [1999]: «Determinantes del plazo de endeudamiento de las empresas españolas», Investigaciones Económicas, 23, 3, pp. 351-392.

Demirgüç-Kunt, A., y VojISLAV MaKsimovic [2002]: «Funding growth in bank-based and market-based financial system: evidence from firm-level data», Journal of Financial Economics, 65, pp. 337-363.

Elyasinal, E.; Lin, G., y Liang, T. [2002]: «The determinants of debt maturity at issuance: a system-based model», Artículo aún no publicado, pp. 1-38.

ERGungor, O. [2004]: «Market vs. Bank-based financial systems: Do investor rights really matter?», Journal of Banking and Finance, 28, pp. 2.869-2.887.

Filatoтchev, I., у Micкiewicz, T. (2002): «Ownership concentration, private benefits of control and debt financing», Artículo no publicado, pp. 1-22.

GALLEGo, F., y LoAYZA, N. [2000]: «Financial structure in Chile: macroeconomic development and microeconomic effects», Banco Central de Chile, Febrero, pp. 1-41.

Gaud, Ph.; Jani, E.; Hoesli, M., y Bender, A. [2005]: «The capital structure of Swiss companies: An empirical analysis using dynamic panel data», European Financial Management, 11, 1, pp. 51-69.

GuJARATI, D. [1990]: Econometría, 2. ${ }^{a}$ Ed., Editorial McGraw-Hill Inc., Bogotá.

Hackbarth, D.; Hennessy, C., y Leland, H. [2004]: «Can the tradeoff theory explain debt structure?», Artículo no publicado, pp. 1-38.

HaRvey, C.; Lins, K., y RopPER, A. [2004]: «The effect of capital structure when expected agency costs are extreme», Journal of Financial Economics, 74, pp. 3-30.

Himmelberg, Ch.; Hubbard, R., y Palia, D. [1999]: «Understanding the determinants of managerial ownership and the link between ownership and performance», Journal of Financial Economics, 53, pp. 353-384.

Houston, J. F., y JAMES, Ch. M. [1996]: «Bank information monopolies and the mix of private and public debt claims», Journal of Finance: 51, pp. 1.863-1.889.

Hovakimian, H.; A.; G., y Tehramian, H. [2004]: «Determinants of target capital structure: The case of dual debt and equity issues», Journal of Financial Economics, 71, pp. 517-540.

J.; T.; у МАкнјА, А. [2005]: «Leverage and the complexity of takeovers», Financial Review,40, pp. 90-112. J.; M., y MeckLING, W. [1976]: «Theory of the firm: managerial behaviour, agency cost and ownership structure», Journal of Financial Economics, 3, 4, pp. 305-360. 
Jensen, M. [1986]: «Agency cost of free cash flow, corporate finance, and takeovers», American Economic Review, 76, 2, pp. 323-329.

- [2004]: «The agency costs of overvalued equity and the current estate of corporate finance», European Financial Management, 10, 4, pp. 549-565.

Johnson, Sh. A. [1997 a)]: «An empirical analysis of the determinants of corporate debt ownership structure», Journal of Financial and Quantitative Analysis, 32, 1, pp. 47-69.

- [1997 b)]: «The effect of bank debt on optimal capital structure», Financial Management, 26, 4, pp. 47-56.

- (2003): «Debt maturity and effects of growth opportunities and liquidity risk on leverage», Review of Financial Studies, 16, 1, pp. 209-236.

Johnson; Simon, y Shleifer, A. [2000]: «Coase and corporate governance in Latin America», Revista ABANTE, 2, 2, pp. 113-131.

Koskela, E., y STENBACKA, R. [2004]: «Agency cost of debt and credit market imperfections: A bargaining approach», Bulletin of Economic Research, 6, 4, pp. 365-377.

Krishnaswami; Sudha; Spindt, P. A., y Subramaniam, V. [1999]: «Information asymmetry, monitoring, and the placement structure of corporate debt», Journal of Financial Economics: 51, pp. 407-434.

LA PoRTA, R.; LóPEZ-DE-Silanes, F., y ShleIFER, A. [1999]: «Corporate ownership around the world», Journal of Finance, 54, pp. 471-517.

La Porta, R.; López-de-Silanes, F.; Shleifer, A., y Vishny, R. [1998]: «Law and finance», Journal of Political Economy: 106, 6, pp. 1.113-1.155.

- [2000]: «Investor protection and corporate governance», Journal of Financial Economics, 58, pp. $3-27$.

LEUZ, Ch.; NANDA, Dh., y WYSocKI, P. D. [2001]: «Investor protection and earnings management: an international comparison», Artículo no publicado.

LEvinE, R. [2002]: «Bank-based or market-based financial systems: which is better?», National Bureau of Economic Research, Working Paper, 9138, pp. 1-27.

LóPEZ, F. [2005]: «Debt ownership structure and legal system: An international analysis», Applied Economics, 37, pp. 355-365.

MAUG, E. [1998]: «Large shareholders as monitors: is there a trade-off between liquidity and control?», Journal of Finance, 53, pp. 65-92.

McConnell, J. J., y Servaes, H. [1990]: «Additional evidence on equity ownership and corporate value», Journal of Financial Economics, 26, pp. 595-612.

McConnelu, J. J., y Servaes, H. [1995]: «Equity ownership and two faces of debt», Journal of Financial Economics, 39, pp. 131-157.

Modigliani, F., y MiLLER, M. [1958]: «The cost of capital, corporation finance and the theory of investment», American Economic Review, 68, 3, pp. 261-297.

MyERS, S. C. [1977]: «Determinants of corporate borrowing», Journal of Financial Economics, 5, pp. 147-175.

— [1984]: «Capital structure puzzle», Journal of Finance, 39, 3, pp. 575-593.

Myers, S., y MaJluF, N. [1984]: «Corporate financing and investment decisions when firms have information that investors don't have», Journal of Financial of Economics, 13, pp. 77-107.

OzKan, A. [2002]: «The determinants of corporate debt maturity: evidence from UK firms», Applied Financial Economics, 12, pp. 19-24.

RodríGuez, J. A. [1997]: «Capital, control y resultados en la gran empresa», Secretariado de Publicaciones e Intercambio Científico, Universidad de Valladolid. Valladolid. 
ShyAm-Sunder, Lakshmi y Stewart C. Myers [1999]: «Testing static tradeoff against pecking order models of capital structure», Journal of Financial Economics, 51, pp. 219-244.

Sogorв, F., y López, J. [2003]: «Pecking order versus trade-off: an empirical approach to the small and medium enterprise capital structure», Artículo no publicado, presentado en la Asociación Científica de Economía y Dirección de Empresa [ACEDE], Salamanca, España.

Sтонs, M. H., у MAUER, D. C. [1996]: «The determinants of corporate debt maturity structure», Journal of Business, 69, 3, pp. 279-312.

TADESSE, S. [2002]: «Financial architecture and economic performance: international evidence», Working Paper, The University of South California.

WINDMEIJER, F. [2000]: «A finite sample correction for the variance of linear two-step GMM estimators», The Institute for Fiscal Studies, Working Paper: 19, pp. 1-25.

WooLDRIDGE, J. [2002]: «Econometric analysis of cross section and panel data», Massachusetts Institute of Technology Press, Cambridge Massachusetts, London. 